\title{
Interaction of race with weight loss and resolution of obesity co-morbidities in patients undergoing laparoscopic roux-en-y gastric bypass (Irygb): an analysis of 83,059 bold database patients
}

\begin{abstract}
Background: Previous studies identified differences by race in the distribution of medical problems associated with morbid obesity. Whether or not outcomes after LRYGB also vary by race is unknown.

Objective: To identify racial variations in weight loss and resolution of obesity comorbidities after LRYGB.

Methods: Data from 83,059 BOLD database LRYGB patients was analyzed retrospectively in five groups: African-American $(n=9,055)$, Caucasian $(n=63,352)$, Hispanic $(n=6,893)$, Asian $(\mathrm{n}=198)$, and Other $(\mathrm{n}=3,561)$.

Results: Weight and BMI were higher in African-Americans versus Caucasians, Hispanics, Other $(12$ months, $\mathrm{p}<0.0001)$. Hypertension persisted increased among African-Americans versus Caucasians, Hispanics, Other through 24 months $(\mathrm{p}<0.01)$. Caucasian cholelithiasis (18 months, $\mathrm{p}<0.05)$, abdominal panniculitis (12 months, $\mathrm{p}<0.01$,) and depression (24 months, $\mathrm{p}<0.05$ ) continued higher than other races. GERD was highest in AfricanAmericans and Caucasians. Dyslipidemia affected Caucasians, African-Americans, and Other most (12 months, $\mathrm{p}<0.05)$. Hispanic depression was lowest ( 24 months, $\mathrm{p}<0.05$ ). Other had highest stress urinary incontinence $(12$ months, $\mathrm{p}<0.05)$. Racial differences in diabetes, liver disease, obstructive sleep apnea, obesity hypoventilation syndrome, gout, back and musculoskeletal pain, leg edema, alcohol use, and non-depression psychological issues were not significant beyond 6 months. Resolution of angina, CHF, pulmonary hypertension, and polycystic ovarian disease did not vary by race.

Conclusions: LRYGB improves obesity weight and co-morbidity outcomes overall, but long-term treatment effects vary by race. African-American weight and hypertension, and African-American/Caucasian GERD, and dyslipidemia resolve least. Caucasian abdominal issues and depression dominate. Racial variations in many obesity co-morbidities disappear by 12 months post-operatively.
\end{abstract}

Volume II Issue 3 - 202 I

\author{
Jeffrey S Emrich, Casey G Sheck, Leon \\ Kushnir, Cristina Nituica, Gus J Slotman \\ Department of Surgery, Inspira Health Network, USA
}

Correspondence: Gus J Slotman, Inspira Health Network, 1505 West Sherman Avenue, Suite BVineland, NJ 08360, USA, Tel 856-64I-8635, Fax 856-64I-8636, Email Slotmang@ihn.org

Received: May 10, 202I | Published: June 07, 2021

\section{Introduction}

The incidence of many medical conditions varies by race. ${ }^{1,2}$ Caucasians have higher gastroesophageal reflux (GERD). ${ }^{3}$ Hypertension, gout, some psychiatric disorders, and back pain are increased among African Americans. ${ }^{2,46}$ Incontinence affects nonHispanic whites more often. ${ }^{7}$ Mortality due to alcohol is highest in Native Americans, followed by African-Americans, Caucasians, and Asians. $^{8}$

The distribution of weight-related medical problems in morbidly obese patients also varies by race. In pre-operative LRYGB patients, Adams et al., ${ }^{1}$ found African-Americans had the highest BMI and rates of asthma and sleep apnea. Asians and African-Americans higher hypertension, Asians highest diabetes, and Caucasians higher ischemic heart disease, cholelithiasis, GERD, liver disease, somatic complaints, and depression. Hispanics had lower rates of hypertension and sleep apnea. ${ }^{1}$

While the clinical characteristics of obese patients vary by race, whether or not race interacts with bariatric surgery outcomes is unknown. The objective of this study was to identify racial variations in weight loss and in the resolution of obesity-related medical problems following LRYGB.

\section{Materials and methods}

With the approval of the Data Access Committee of the Surgical Review Corporation and of the Institutional Review Board of Our Lady of Lourdes Medical Center, Camden, NJ data from 83,059 Surgical Review Corporation Bariatric Outcomes Longitudinal Database (BOLD) LRYGB patients was analyzed retrospectively in five groups: African-American $(\mathrm{n}=9,055)$, Caucasian $(\mathrm{n}=63,352)$, Hispanic $(\mathrm{n}=6,893)$, Asian $(\mathrm{n}=198)$, and Other (Pacific Islander, Native American, or $>1$ race listed in BOLD; $n=3,561) .{ }^{9}$

Data included age, weight, BMI, sex, race and the percentage frequency of hypertension (HTN), angina, congestive heart failure (CHF), deep vein thrombosis/pulmonary embolism (DVT/ $\mathrm{PE}$ ), ischemic heart disease, peripheral vascular disease (PVD), pulmonary hypertension (PHT), obstructive sleep apnea (OSA), obesity hypoventilation syndrome (OHS), asthma, abdominal hernia, panniculitis, cholelithiasis, gastroesophageal reflux disease (GERD), liver disease, stress urinary incontinence (SUI), diabetes mellitus, 
gout, hyperlipidemia, irregular menses, polycystic ovarian syndrome (PCOS), pseudotumor cerebri, back pain, fibromyalgia, lower extremity edema, musculoskeletal pain, mental health diagnoses (MHD), disability, depression, psychologic impairment, alcohol, substance, and tobacco use. Although BOLD recorded several degrees of disease severity for each obesity co-morbidity, to secure adequate analytic statistical power, these gradations were grouped together as a dichotomous present/absent designation.

Statistical analysis used General Linear Models that included baseline and post-operative data, and were modified for binomial distribution of dichotomous variables. Continuous variables were analyzed using an ANOVA with treatment in the model. Pair-wise comparisons were performed on the least squares means of the treatments calculated from the ANOVA model to find differences in the treatment groups.

\section{Results}

For African-Americans, Caucasians, Hispanics, Asians, and Other, respectively, age was $43+-10,47+-8,48+-8,46+-8,47+-8(\mathrm{p}<0.0001)$, and female/male $\%$ was $86 / 14,78 / 22,78 / 22,73 / 27,81 / 19$ ( $<<0.0001)$. The numbers of evaluable patients at pre-operative baseline, and at 2 , 6, 12, 18, 24 months after LYRGB were: African-Americans - 9,055, 7,439, 4,285, 2,089, 599, 405; Caucasians - 63,352, 48,986, 36,752, 21,362, 5,490, 4,469; Hispanics - 6,893, 4,091, 3,503, 1,659, 426, 273; Asians - 198, 137, 102, 46, 11, 4; Other - 3,561, 5,213, 1,854, $975,300,211$

Weight, weight loss and BMI by race are listed in Table 1. Weight and BMI were highest through 24 months in African-Americans and Asians.

Table I Weight and BMI after LRYGB by race

\begin{tabular}{|c|c|c|c|c|c|c|}
\hline $\begin{array}{l}\text { Weight } \\
\text { (kg) }\end{array}$ & Baseline & 2 months & 6 months & 12 months & 18 months & 24 months \\
\hline \multirow[t]{2}{*}{$\begin{array}{l}\text { African- } \\
\text { American }\end{array}$} & 139 & 123 & 105 & 94 & 92 & 90 \\
\hline & -29 & -27 & -24 & -22 & -21 & -20 \\
\hline \multirow[t]{2}{*}{ Caucasian } & 133 & 115 & 90 & 86 & 83 & 84 \\
\hline & -27 & -26 & -21 & -20 & -20 & -20 \\
\hline \multirow[t]{2}{*}{ Hispanic } & 129 & 113 & 95 & 85 & 82 & 83 \\
\hline & -27 & -25 & -21 & -19 & -19 & -19 \\
\hline \multirow[t]{2}{*}{ Asian } & 124 & 109 & 95 & 87 & 87 & 95 \\
\hline & -27 & -23 & -20 & -21 & -28 & -41 \\
\hline \multirow[t]{2}{*}{ Other } & $|3|$ & 114 & 95 & 86 & 83 & 86 \\
\hline & -27 & -24 & -21 & -20 & -18 & -21 \\
\hline \multirow[t]{2}{*}{$p$-value } & $A A$ vs $A l l=<0.01$ & AA vs $A l l=<0.01$ & $\mathrm{AA}$ vs $\mathrm{O}, \mathrm{C}, \mathrm{H}=<0.000 \mathrm{I}$ & AA vs $\mathrm{O}, \mathrm{C}, \mathrm{H}=<0.000 \mathrm{I}$ & AA vs $\mathrm{O}, \mathrm{C}, \mathrm{H}=<0.00 \mathrm{I}$ & $\mathrm{AA}$ vs $\mathrm{C}, \mathrm{H}=<0.05$ \\
\hline & C vs $\mathrm{O}, \mathrm{H}=<0.0 \mathrm{I}$ & $\mathrm{C}$ vs $\mathrm{O}, \mathrm{H}=<0.000 \mathrm{I}$ & C vs $\mathrm{O}, \mathrm{H}=<0.000 \mathrm{I}$ & C vs $\mathrm{O}, \mathrm{H}=<0.000 \mathrm{I}$ & $\mathrm{C}, \mathrm{O}, \mathrm{H}$ vs $\mathrm{A}=<0.000 \mathrm{I}$ & $C$ vs $\mathrm{O}=<0.05$ \\
\hline BMI & Baseline & 2 months & 6 months & 12 months & 18 months & 24 months \\
\hline \multirow[t]{2}{*}{$\begin{array}{l}\text { African- } \\
\text { American }\end{array}$} & 50 & 44 & 38 & 34 & 33 & 33 \\
\hline & -9 & -8 & -8 & -7 & -7 & -7 \\
\hline \multirow[t]{2}{*}{ Caucasian } & 47 & 41 & 34 & 31 & 30 & 30 \\
\hline & -8 & -7 & -6 & -6 & -6 & -6 \\
\hline \multirow[t]{2}{*}{ Hispanic } & 48 & 41 & 35 & 32 & 30 & 31 \\
\hline & -8 & -7 & -7 & -6 & -6 & -6 \\
\hline \multirow[t]{2}{*}{ Asian } & 46 & 40 & 35 & 32 & 33 & 35 \\
\hline & -8 & -7 & -6 & -6 & -7 & -12 \\
\hline \multirow[t]{2}{*}{ Other } & 47 & 42 & 35 & 31 & 30 & 31 \\
\hline & -9 & -8 & -7 & -6 & -6 & -7 \\
\hline \multirow[t]{3}{*}{$\mathrm{p}$-value } & $<0.0001$ & AA vs $\mathrm{All}=<0.02$ & $\mathrm{AA}$ vs $\mathrm{C}, \mathrm{O}, \mathrm{H}=<0.00 \mathrm{I}$ & AA vs C.O.H = <0.000I; & AA vs C.O.H = <0.0I; & $A A$ vs $C . H=<0.01$ \\
\hline & & $\mathrm{C}$ vs $\mathrm{O}, \mathrm{H}=<0.000 \mathrm{I}$ & $\mathrm{C}$ vs $\mathrm{O}, \mathrm{H}, \mathrm{A}=<0.0 \mathrm{I}$ & $\mathrm{C}$ vs $\mathrm{O}, \mathrm{H}, \mathrm{A}=<0.0 \mathrm{I}$ & A vs $\mathrm{C}, \mathrm{O}, \mathrm{H}=<0.05$ & $\mathrm{C}$ vs $\mathrm{O}=<0.05$ \\
\hline & & & A vs $\mathrm{O}, \mathrm{H}=<0.05$ & A vs $\mathrm{H}=<0.05$ & & \\
\hline
\end{tabular}

AA, African American; C, Caucasian; H, Hispanic; A, Asian; O, other 
Cardio-Pulmonary co-morbidities by race are displayed in Table 2. CHF, PVD, PHT, and asthma outcomes did not vary by race. Through 24 months, Aric-American hypertension persisted higher than Caucasians, Hispanics, and Other $(\mathrm{p}<0.01)$. Hypertension was lowest among Hispanics. Asian hypertension decreased early, and then rose to pre-operative levels at 24 months. OSA was lowest among Hispanics and Other at 2 and 6 months. Angina and OHS varied by race only at 2 months.

Table 2 Cardio-pulmonary comorbidities after laparoscopic roux-en-Y gastric bypass by race

\begin{tabular}{|c|c|c|c|c|c|}
\hline Months & Race & HTN & OSA & ANGINA & OHS \\
\hline \multirow{6}{*}{ Baseline } & African American & 65.46 & 45.81 & 3.16 & 1.54 \\
\hline & Caucasian & 61.18 & 44.41 & 3.01 & 2 \\
\hline & Hispanic & 50.18 & 40.5 & 1.86 & 1.26 \\
\hline & Asian & 65.66 & 45.45 & 1.52 & 1.52 \\
\hline & Other & 53.27 & 39.12 & 1.91 & 1.85 \\
\hline & $P$ & $p<0.000 I$ & $<0.0001$ & $<0.0001$ & $<0.0001$ \\
\hline \multirow{6}{*}{2} & African American & 56.36 & 39.89 & 2.46 & 1.46 \\
\hline & Caucasian & 50.28 & 43.09 & 2.39 & 1.68 \\
\hline & Hispanic & 39.97 & 34.33 & 1.39 & 0.99 \\
\hline & Asian & 54.64 & 39.18 & 1.55 & 1.03 \\
\hline & Other & 44.57 & 33.65 & 1.49 & 1.47 \\
\hline & $P$ & $\begin{array}{l}\text { AA vC, } \mathrm{O}, \mathrm{H}<0.00 \mathrm{I} \\
\mathrm{H} \vee \mathrm{C}, \mathrm{O}<0.00 \mathrm{I}\end{array}$ & $\mathrm{H}$ vs $\mathrm{AA}, \mathrm{C}=<0.0 \mathrm{I}$ & $\mathrm{C}$ vs $\mathrm{H}=<0.05$ & AA vs $\mathrm{C}, \mathrm{H}=<0.05$ \\
\hline \multirow{6}{*}{6} & African American & 49.54 & 32.16 & 2.1 & 1.33 \\
\hline & Caucasian & 39.5 & 33.09 & 2.01 & $\mathrm{I} .44$ \\
\hline & Hispanic & 31.17 & 27.03 & 1.03 & 0.86 \\
\hline & Asian & 41.18 & 27.45 & 1.96 & 0.98 \\
\hline & Other & 37.32 & 26.16 & 0.97 & 0.97 \\
\hline & $P$ & $\begin{array}{l}A A \vee C, O, H<0.000 I \\
H \vee C, O<0.00 I\end{array}$ & $A A$ vs $C=<0.05$ & NS & NS \\
\hline \multirow{6}{*}{12} & African American & 46.43 & 26.76 & 1.77 & 1.63 \\
\hline & Caucasian & 32.75 & 25.77 & 1.86 & 1.34 \\
\hline & Hispanic & 25.8 & $|8.5|$ & 0.6 & 0.84 \\
\hline & Asian & 32.61 & 10.87 & 2.17 & $(0 / 46)$ \\
\hline & Other & 30.67 & 21.95 & 0.82 & 0.92 \\
\hline & $P$ & NS & NS & NS & NS \\
\hline \multirow{6}{*}{18} & African American & 43.07 & 21.95 & 2.5 & 1.17 \\
\hline & Caucasian & 29.36 & 22.33 & 1.69 & 1.38 \\
\hline & Hispanic & 24.41 & 19.01 & 0.7 & 1.17 \\
\hline & Asian & 63.64 & 27.27 & 0 & $(0 / 11)$ \\
\hline & Other & 29 & 18.67 & 1.67 & 1.33 \\
\hline & $P$ & $\mathrm{AA} \vee \mathrm{C}, \mathrm{O}, \mathrm{H}<0.00 \mathrm{I}$ & NS & NS & NS \\
\hline \multirow{6}{*}{24} & African American & 43.95 & 21.73 & 2.22 & 2.22 \\
\hline & Caucasian & 27.57 & 20.09 & 1.57 & 1.36 \\
\hline & Hispanic & 23.08 & 19.01 & 0.73 & 1.47 \\
\hline & Asian & & 25 & 0 & $(0 / 4)$ \\
\hline & Other & 28.44 & 18.01 & 1.9 & $(0 / 211)$ \\
\hline & $P$ & $\mathrm{AA} \vee \mathrm{C}, \mathrm{O}, \mathrm{H}<0.0 \mathrm{I}$ & NS & NS & NS \\
\hline
\end{tabular}


Metabolic and endocrine co-morbidities by race are tabulated in Table 3. Diabetes varied by race through six months. Dyslipidemia was lowest among Hispanics and Asians, and highest in Caucasians and Other through 12 months $(\mathrm{p}<0.05)$. Abdominal and Hepatobiliary co-morbidities by race are seen in Table 4. Cholelithiasis persisted essentially at baseline levels, Caucasians highest. Abdominal panniculitis was highest iCaucasians and lowest Asians and Hispanics through 12 months. GERD was lower through 18 months in Asians and Hispanics, and highest in African-Americans and Caucasians $(\mathrm{p}<0.05)$. Liver disease was highest in Asians and Caucasians through 6 months, and nearly doubled among Asians. Through 12 months, SUI was lowest among Asians and Hispanics and highest in Other $(\mathrm{p}<0.05)$.

Table 3 Metabolic and endocrine comorbidities after laparoscopic roux-en-Y gastric bypass by race

\begin{tabular}{|c|c|c|c|c|}
\hline Months & Race & DM & Gout & Dyslipidemia \\
\hline \multirow{6}{*}{ Baseline } & $\begin{array}{l}\text { African } \\
\text { American }\end{array}$ & 35.94 & 4.79 & 30.86 \\
\hline & Caucasian & 39.52 & 3.85 & 46.87 \\
\hline & Hispanic & 38.1 & 1.7 & 31.37 \\
\hline & Asian & 52.02 & 4.55 & 52.02 \\
\hline & Other & 37.01 & 2.92 & 36.06 \\
\hline & $P$ & $<0.0001$ & $<0.0001$ & $<0.0001$ \\
\hline \multirow{6}{*}{2} & $\begin{array}{l}\text { African } \\
\text { American }\end{array}$ & 26.61 & 3.85 & 27.24 \\
\hline & Caucasian & 27.48 & 3.38 & 41.16 \\
\hline & Hispanic & 28.61 & 1.51 & 27.19 \\
\hline & Asian & 39.18 & 4.12 & 46.39 \\
\hline & Other & 26.24 & 2.76 & 32.47 \\
\hline & $P$ & $\begin{array}{l}\text { CvsAA, } \mathrm{H}=<0.000 \mathrm{I} \\
\text { O vs } \mathrm{H}=<0.05\end{array}$ & AA vs $C, O=<0.05$ & $\mathrm{H}$ vs $\mathrm{C}, \mathrm{O}=<0.0 \mathrm{I}$ \\
\hline \multirow{6}{*}{6} & $\begin{array}{l}\text { African } \\
\text { American }\end{array}$ & 19.6 & 3.29 & 22.52 \\
\hline & Caucasian & 19.32 & 2.95 & 34.06 \\
\hline & Hispanic & 20.24 & 1.14 & 21.1 \\
\hline & Asian & 23.53 & 0.98 & 39.22 \\
\hline & Other & 19.42 & 1.78 & 29.13 \\
\hline & $P$ & $\begin{array}{l}C \text { vs } A A=<0.01 ; C \text { vs } \\
H=<0.05\end{array}$ & NS & $\begin{array}{l}\mathrm{H} \text { vs } \mathrm{AA}, \mathrm{O}, \mathrm{C}=<0.00 \mathrm{I} \\
\mathrm{H} \text { vs } \mathrm{A}<0.05 ; \mathrm{C} \text { vs } \\
\mathrm{H}=<0.000 \mathrm{I} ; \\
\mathrm{C} \text { vs } \mathrm{O}=<0.05\end{array}$ \\
\hline \multirow{6}{*}{12} & $\begin{array}{l}\text { African } \\
\text { American }\end{array}$ & 16.61 & 3.06 & 20.39 \\
\hline & Caucasian & 14.36 & 2.74 & 28.15 \\
\hline & Hispanic & 15.43 & 1.27 & 15.73 \\
\hline & Asian & 15.22 & $(0 / 46)$ & 19.57 \\
\hline & Other & 13.85 & 2.15 & 27.18 \\
\hline & $P$ & NS & NS & $\begin{array}{l}\mathrm{A} \text { vs } \mathrm{AA}, \mathrm{C}, \mathrm{O}=<0.05 \\
\mathrm{H} \text { vs } \mathrm{AA}, \mathrm{C}, \mathrm{O}=<0.000 \mathrm{I}\end{array}$ \\
\hline \multirow{6}{*}{18} & $\begin{array}{l}\text { African } \\
\text { American }\end{array}$ & 12.35 & 1.67 & 18.86 \\
\hline & Caucasian & 12.55 & 2.64 & 25.99 \\
\hline & Hispanic & 15.26 & 0.47 & 15.96 \\
\hline & Asian & 27.27 & $(0 / I I)$ & 36.36 \\
\hline & Other & 11.67 & 1.33 & 26 \\
\hline & $P$ & NS & NS & NS \\
\hline
\end{tabular}

Citation: Emrich JS, Sheck CG, Kushnir L, et al. Interaction of race with weight loss and resolution of obesity co-morbidities in patients undergoing laparoscopic roux-en-y gastric bypass (Irygb): an analysis of 83,059 bold database patients. Adv Obes Weight Manag Control. 202 I; I I(3):76-84. 


\begin{tabular}{lllll}
\multicolumn{2}{l}{ Table Continued... } & & Gout & Dyslipidemia \\
\hline Months & Race & DM & 1.48 & 20.49 \\
24 & African & 14.32 & 2.24 & 23.81 \\
& American & & 0.73 & 15.75 \\
& Caucasian & 14.32 & $(0 / 4)$ & $(0 / 4)$ \\
& Hispanic & 11.72 & 0.47 & 28.44 \\
& Asian & $(0 / 4)$ & NS & NS
\end{tabular}

DM, diabetes mellitus; AA, African American; C, Caucasian; H, Hispanic; A, Asian; O, other

Table 4 Abdominal and hepatobiliary comorbidities after laparoscopic roux-En-Y gastric bypass by race

\begin{tabular}{|c|c|c|c|c|c|c|}
\hline Months & Race & Cholelithiasis & $\begin{array}{l}\text { Abdominal } \\
\text { Panniculitis }\end{array}$ & GERD & Liver Disease & Abdominal Hernia \\
\hline \multirow{6}{*}{ Baseline } & $\begin{array}{l}\text { African } \\
\text { American }\end{array}$ & 14.46 & 4.61 & 43.43 & 3.96 & 4.61 \\
\hline & Caucasian & 23.16 & 8.51 & 52.15 & 7.79 & 8.51 \\
\hline & Hispanic & 18.89 & 5.06 & 39.78 & 6.69 & 5.06 \\
\hline & Asian & 12.12 & 5.56 & 35.35 & 6.57 & 5.56 \\
\hline & Other & 17.13 & 5.45 & 42.94 & 5.56 & 5.45 \\
\hline & $P$ & $<0.0001$ & $<0.001$ & $<0.0001$ & $<0.001$ & $<0.001$ \\
\hline \multirow{6}{*}{2} & $\begin{array}{l}\text { African } \\
\text { American }\end{array}$ & 14.4 & 4.48 & 33.78 & 3.74 & 4.48 \\
\hline & Caucasian & 22.96 & 8.15 & 39.45 & 7.64 & 8.15 \\
\hline & Hispanic & 18.01 & 4.65 & 30.26 & 6.36 & 4.65 \\
\hline & Asian & 10.82 & 6.7 & 25.77 & 7.22 & 6.7 \\
\hline & Other & 16.7 & 4.83 & 33.85 & 4.89 & 4.83 \\
\hline & $P$ & $\begin{array}{l}A A \text { vs } A L L=<0.00 I \\
C \text { vs } O, A, H=<0.00 I \\
A \text { vs } O, H=<0.04\end{array}$ & $\begin{array}{l}C \text { vs } \mathrm{AA}, \mathrm{H}, \mathrm{O}=<0.05 \\
\mathrm{~A} \text { vs } \mathrm{O}=<0.05\end{array}$ & $\mathrm{O}$ vs $\mathrm{H}=<0.05$ & $\begin{array}{l}C \text { vs } A A=<0.000 I \\
C \text { vs } H=<0.05 ; \text { Cvs } \\
O=<0.01\end{array}$ & $\begin{array}{l}C \text { vs } A A, H, O=<0.05 ; \\
A \text { vs } O=<0.05\end{array}$ \\
\hline \multirow{6}{*}{6} & $\begin{array}{l}\text { African } \\
\text { American }\end{array}$ & 15.64 & 4.97 & 26.49 & 3.62 & 4.97 \\
\hline & Caucasian & 23.1 & 8.5 & 29.05 & 7.27 & 8.5 \\
\hline & Hispanic & 18.36 & 4.97 & 22.61 & 5.54 & 4.97 \\
\hline & Asian & 9.8 & 4.9 & 18.63 & 8.82 & 4.9 \\
\hline & Other & 17.64 & 5.39 & 26.86 & 4.37 & 5.39 \\
\hline & $P$ & $\begin{array}{l}\mathrm{AA} \text { vs } \mathrm{ALL}=<0.00 \mathrm{I} \\
\mathrm{C} \text { vs } \mathrm{O}, \mathrm{A}, \mathrm{H}=<0.00 \mathrm{I} \\
\mathrm{A} \text { vs } \mathrm{O}, \mathrm{H}=<0.04\end{array}$ & $\mathrm{H}$ vs $\mathrm{AA}, \mathrm{C}, \mathrm{O}=<0.05$ & $\begin{array}{l}C \text { vs } A A, O=<0.05 \\
H \text { vs } A A, O=<0.05\end{array}$ & $\begin{array}{l}C \text { vs } A A, A, H=<0.01 \\
A \vee A A, O=<0.01\end{array}$ & $\mathrm{H}$ vs $\mathrm{AA}, \mathrm{C}, \mathrm{O}=<0.05$ \\
\hline \multirow{6}{*}{12} & $\begin{array}{l}\text { African } \\
\text { American }\end{array}$ & $|5.5|$ & 5.84 & 24.61 & 3.59 & 5.84 \\
\hline & Caucasian & 23.99 & 9.11 & 24.79 & 6.9 & 9.11 \\
\hline & Hispanic & 18.02 & 4.22 & 18.63 & 5.24 & 4.22 \\
\hline & Asian & 13.04 & 2.17 & 13.04 & 13.04 & 2.17 \\
\hline & Other & 17.44 & 6.05 & 22.15 & 4.41 & 6.05 \\
\hline & $P$ & $\begin{array}{l}\mathrm{AA} \text { vs } \mathrm{ALL}=<0.00 \mathrm{I} ; \mathrm{C} \\
\text { vs } \mathrm{O}, \mathrm{A}, \mathrm{H}=<0.00 \mathrm{I} ; \\
\mathrm{A} \text { vs } \mathrm{O}, \mathrm{H}=<0.04\end{array}$ & $\mathrm{H}$ vs $\mathrm{AA}, \mathrm{C}, \mathrm{O}=<0.05$ & $\begin{array}{l}\text { AA vs } C, H \\
=<0.000 I\end{array}$ & $\begin{array}{l}\text { ALL NON- } \\
\text { SIGNIFICANT }\end{array}$ & $\mathrm{H}$ vs $\mathrm{AA}, \mathrm{C}, \mathrm{O}=<0.05$ \\
\hline
\end{tabular}


Table Continued..

\begin{tabular}{|c|c|c|c|c|c|c|}
\hline Months & Race & Cholelithiasis & $\begin{array}{l}\text { Abdominal } \\
\text { Panniculitis }\end{array}$ & GERD & Liver Disease & Abdominal Hernia \\
\hline \multirow{5}{*}{18} & $\begin{array}{l}\text { African } \\
\text { American }\end{array}$ & 15.03 & 6.51 & 22.2 & 5.68 & 6.51 \\
\hline & Caucasian & 24.68 & 10.77 & 24.54 & 7.25 & 10.77 \\
\hline & Hispanic & 19.01 & 6.81 & 15.49 & 4.46 & 6.81 \\
\hline & Other & 18.67 & 3 & 21 & 3.67 & 3 \\
\hline & $P$ & $\begin{array}{l}\text { AA vs } A L L=<0.00 I ; C \\
\text { vs } O, A, H=<0.00 I ; \\
\text { A vs } O, H=<0.04\end{array}$ & All non-significant & $\mathrm{H}$ vs $\mathrm{C}, \mathrm{AA}=<0.05$ & All non-significant & All non-significant \\
\hline \multirow{5}{*}{24} & $\begin{array}{l}\text { African } \\
\text { American }\end{array}$ & $|4.8|$ & 4.2 & 21.48 & 4.44 & 4.2 \\
\hline & Hispanic & 15.38 & 5.86 & 16.48 & 3.66 & 5.86 \\
\hline & Asian & $(0 / 4)$ & $(0 / 4)$ & $(0 / 4)$ & $(0 / 4)$ & $(0 / 4)$ \\
\hline & Other & 18.01 & 3.32 & 21.33 & 2.84 & 3.32 \\
\hline & $P$ & $\mathrm{O}$ vs $\mathrm{H}=<0.04$ & All non-significant & All non-significant & All non-significant & All non-significant \\
\hline
\end{tabular}

GERD, gastroesophageal reflux disease;AA, African American; C, Caucasian; H, Hispanic; A, Asian; O, other

Somatic co-morbidities by race are listed in Table 5. Back pain was lowest in Hispanics versus Caucasians, African-Americans, and Other $(\mathrm{p}<0.05)$. Through 6 months, lower extremity edema and musculoskeletal pain were lowest in Hispanics and Asians $(p<0.05)$.

Psychological/Behavioral co-morbidities by race are displayed in Table 4. Mental health diagnosis was highest in Caucasians and Asians at 2 and 6 months, but changed little from pre-operative in any group. Post-operative depression was lower among AfricanAmericans, Hispanics, and Asian compared with Caucasians and the Other group, and persisted lowest in Hispanics and highest for Caucasians through 24 months. Psychological impairment was lowest in African-Americans through 6 months, and even lower among Hispanics and Asians at 18 months, and was consistently highest for Caucasians. Alcohol use varied only through 6 months.

Table 5 Musculoskelatal comorbidities after laparoscopic roux-En-Y gastric bypass by race

\begin{tabular}{|c|c|c|c|c|}
\hline Months & Race & Back pain & $\begin{array}{l}\text { Lower extremity } \\
\text { edema }\end{array}$ & Musculoskelatal pain \\
\hline \multirow{6}{*}{ Baseline } & $\begin{array}{l}\text { African } \\
\text { American }\end{array}$ & 44.01 & 26.2 & 26.17 \\
\hline & Caucasian & 52.09 & 32.2 & 32.24 \\
\hline & Hispanic & 42.01 & 20.5 & 20.53 \\
\hline & Asian & 42.93 & 24.8 & 24.75 \\
\hline & Other & 41.03 & 23 & 22.97 \\
\hline & $P$ & $<0.000$ I & $<0.000$ I & $<0.0001$ \\
\hline \multirow{6}{*}{2} & $\begin{array}{l}\text { African } \\
\text { American }\end{array}$ & 38.55 & 20.55 & 33.89 \\
\hline & Caucasian & 45.17 & 23.58 & 41.35 \\
\hline & Hispanic & 35.68 & 15.36 & 27.69 \\
\hline & Asian & 37.63 & 18.04 & 31.44 \\
\hline & Other & 36.29 & 17.41 & 33.16 \\
\hline & $P$ & $\mathrm{H}$ vs $\mathrm{O}, \mathrm{C}, \mathrm{AA}=<0.05$ & $A A$ vs $C, H=<0.01$ & $\begin{array}{l}\text { C vs } A A, H=<0.000 I \\
O \text { vs } H=<0.05\end{array}$ \\
\hline
\end{tabular}


Table Continued..

\begin{tabular}{|c|c|c|c|c|}
\hline Months & Race & Back pain & $\begin{array}{l}\text { Lower extremity } \\
\text { edema }\end{array}$ & Musculoskelatal pain \\
\hline \multirow{6}{*}{6} & $\begin{array}{l}\text { African } \\
\text { American }\end{array}$ & 33.37 & 17.43 & 29.87 \\
\hline & Caucasian & 37.61 & 18.12 & 34.77 \\
\hline & Hispanic & 29.03 & 12.3 & 23.52 \\
\hline & Asian & $29.4 I$ & 8.82 & 20.59 \\
\hline & Other & 31.07 & 13.43 & 28.21 \\
\hline & $P$ & $\mathrm{H}$ vs $\mathrm{O}, \mathrm{C}, \mathrm{AA}=<0.0 \mathrm{I}$ & AA vs $\mathrm{C}, \mathrm{O}, \mathrm{H}=<0.0 \mathrm{I}$ & $\mathrm{H}$ vs $\mathrm{C}, \mathrm{O}=<0.05$ \\
\hline \multirow{6}{*}{12} & $\begin{array}{l}\text { African } \\
\text { American }\end{array}$ & 31.5 & 16.75 & 29.01 \\
\hline & Caucasian & 33.39 & 15.47 & 30.69 \\
\hline & Hispanic & 25.68 & 9.76 & 19.53 \\
\hline & Asian & 34.78 & 10.87 & 17.39 \\
\hline & Other & 29.23 & 12.72 & 24.92 \\
\hline & $P$ & All non- significant & All non-significant & All non-significant \\
\hline \multirow{6}{*}{18} & $\begin{array}{l}\text { African } \\
\text { American }\end{array}$ & 29.05 & 17.2 & 26.38 \\
\hline & Caucasian & 30.27 & 14.55 & 27.8 \\
\hline & Hispanic & 19.72 & 9.62 & 18.08 \\
\hline & Asian & 18.18 & 9.09 & 27.27 \\
\hline & Other & 29.33 & 11.67 & 25 \\
\hline & $P$ & $\mathrm{H}$ vs $\mathrm{AA}, \mathrm{O}, \mathrm{C}=<0.05$ & All non-significant & All non-significant \\
\hline \multirow{6}{*}{24} & $\begin{array}{l}\text { African } \\
\text { American }\end{array}$ & 27.9 & 15.31 & 26.42 \\
\hline & Caucasian & 29.85 & 13.29 & 26.36 \\
\hline & Hispanic & 24.91 & 8.06 & 18.68 \\
\hline & Asian & 24.91 & 0 & $n=4$ \\
\hline & Other & 32.23 & 10.43 & 29.86 \\
\hline & $P$ & O vs $\mathrm{H}, \mathrm{C}, \mathrm{AA}=<0.05$ & All non-significant & All non-significant \\
\hline
\end{tabular}

Following LRYGB African-Americans had highest hypertension, and angina, and the lowest psychological impairment, MHD, and liver disease. Caucasians were highest in OSA, OHS, abdominal panniculitis, cholelithiasis, GERD, liver disease (2 months), SUI (2 months), dyslipidemia (12 months), back pain, lower extremity edema, musculoskeletal pain, depression, and psychological impairment $(n=14)$, second highest in angina, MHD, and alcohol consumption, and lowest in none. Hispanics did not rank highest in any co-morbidity, and had lowest hypertension, angina, OHS, abdominal panniculitis, gout, dyslipidemia, back pain, lower extremity edema, musculoskeletal pain (2 months), depression, psychological impairment and alcohol consumption (6 months) $(\mathrm{n}=13)$. Asians had the highest prevalence of liver disease, diabetes, gout, dyslipidemia, MHD, and alcohol consumption $(\mathrm{n}=6)$, and lowest abdominal panniculitis, cholelithiasis, GERD, stress urinary incontinence, back pain (18 months), lower extremity edema (6 months), musculoskeletal pain (6 months), and depression $(\mathrm{n}=8)$. Other group patients were highest in no category and had the lowest OSA, diabetes, alcohol consumption (2 months) $(n=3)$.
Post-operative outcomes did not vary by race for CHF, pulmonary hypertension, asthma, peripheral vascular disease, PCOS, pseudotumor cerebri, impaired functional status, and substance abuse.

\section{Discussion}

The results of this study identify statistically and clinically significant variations by race in weight, BMI, and the resolution of obesity co-morbidities following LRYGB. Post-LRYGB weight loss occurred less rapidly among African-Americans and Asians compared with Caucasians, Hispanics, and Other. Hypertension persisted at higher rates among African-Americans than in the other groups. Obstructive sleep apnea was more prevalent among Caucasians up to six months following LRYGB, suggesting slower OSA resolution in that group. Abdominal conditions affected Caucasians most frequently. Alcohol consumption and liver disease were highest for Asians, among whom liver disease doubled. Diabetes mellitus resolved significantly in all racial groups, varying by race only up to 6 months. Dyslipidemia reduced more in Hispanics and Asians than in 
the other race groups through 12 months. Somatic complaints persisted higher among African-Americans and Caucasians.Mental health issues affected Caucasians and Asians most often. Angina, CHF, PHT, asthma, OHS, PVD, abdominal hernia, gout, PCOS, pseudotumor cerebri, and impaired functional status did not vary by race beyond 2 months after LRYGB. Overall, Caucasians fared worst in resolving obesity co-morbidities and Hispanics benefitted most from LRYGB. Our review of the literature indicates that the significant variations by race of clinical outcomes after LRYGB observed here have not been reported previously in a patient population this large, and are important findings of this study.

After LRYGB, weight and BMI decreased significantly more rapidly among Caucasians, Hispanics and Other than in AfricanAmericans and Asians through 24 months. This data confirms the findings of Sudan et al that African American weight loss and decline in BMI following LRYGB were less than for Caucasians or Hispanics, and adds Asians as a second ethnic category associated with inferior body mass reduction results. ${ }^{10}$ Previous single institution investigations have reported decreased weight loss and BMI reduction in African-Americans versus Caucasians. ${ }^{1,11-17}$ In contrast, Coleman and co-authors found greater reduction in weight and BMI among African-Americans than in non-Hispanic white patients. ${ }^{16}$ Our results further identified significantly impaired weight and BMI response to LRYGB among Asians, which is a new finding. The underlying causes of racial variation in post-LRYGB weight and BMI are not clear from the data.

Diabetes varied by race, highest among Asians and Caucasians lower in African-Americans and Hispanics up to 6 months. Consistent with previous reports, diabetes resolved effectively among all racial groups in our study. ${ }^{13,17}$ In contrast, Coleman and co-workers found that postoperative resolution of diabetes and metabolic syndrome was less likely to occur among Hispanics and non-Hispanic blacks in a recent study. ${ }^{16}$ The body of literature indicates that obesity-induced Type II diabetes mellitus resolves well in all racial groups following LRYGB.

Through 24 months, hypertension persisted at higher rates among African-Americans than other cohorts, and continued higher than Caucasians at 24 months. This is an important finding, as prior studies have not identified such statistically and clinically significant variations by race in the resolution of hypertension after LRYGB. . $^{13,17,18,22}$ Consistent with the known increased prevalence of hypertension in the general African-American population, Adams observed the highest rates of hypertension in Asians and African-Americans among morbidly obese patients pre-operative for LRYGB. ${ }^{1}$ The results here indicate that the increased African-American propensity for hypertension extends to impaired high blood pressure resolution following LRYGB. This advance knowledge should give bariatric surgeons an increased index of suspicion for persistent hypertension among African-Americans after LRYGB.

Data in the literature regarding remission of OSA following bariatric surgery has been conflicting. Weight loss is an important predictor for resolution, but other independent predictors, for example LRYGB compared to LAGB, have been reported..$^{15}$ OSA was more prevalent among Caucasians in this study and, through six months following LRYGB, indicating slower resolution in this demographic. These findings confirm previous reports of OSA resolution lower in Caucasians and Hispanics than African Americans. ${ }^{13}$

Abdominal problems, including panniculitis, cholelithiasis, GERD, and stress urinary incontinence, persisted and/or developed most frequently among Caucasians in the present study. Caucasian cholelithiasis was highest through 18 months. Conversely, panniculitis was lowest for Hispanics. African American SUI was reduced compared with Caucasians and Hispanics. GERD was less common in Hispanics and African-Americans compared to Caucasians. Abdominal comorbidities overall persisted among Caucasians to the greatest extent in our study, correlating with the published literature. ${ }^{10}$

Following LRYGB, liver disease nearly doubled among Asians, while it decreased in the comparator groups. Interestingly, although not statistically significant, Asian alcohol use was also consistently highest. High Asian alcohol use, specifically in Chinese individuals has been reported. ${ }^{8}$ Higher rates of liver disease among the Asian population may be a direct correlate of greater alcohol consumption among Asians, reported here and substantiated in the literature. ${ }^{17}$ However, it is important to note that liver disease was defined clinically in the BOLD database, as biopsies were not required to confirm the diagnosis. Nevertheless, the findings here are important contributions to our understanding of liver disease after LRYGB.

Our data indicates that dyslipidemia resolves at higher rates following LRYGB among Hispanics and Asians than AfricanAmericans and Caucasians. Prior studies have not demonstrated such racial variation in the resolution of hyperlipidemia at 1-year followup after bariatric surgery. ${ }^{17}$ Although many have discontinued lipidlowering medications following bariatric procedures,${ }^{19}$ the mechanisms underlying the racial variations in dyslipidemia responses observed in this study are not clear from the data. Somatic complaints persisted higher among African Americans and Caucasians in our study. The pathophysiology underlying this finding is unknown. LRYGB significant improved of self-reported complaints of back and lower extremity pain. ${ }^{20}$ However, variation in resolution of somatic between racial groups has not been reported previously. Thus, the finding here of higher somatic co-morbidities among African Americans and Caucasians is an important new contribution to the literature.

In this investigation, Caucasians consistently experienced the highest rates of depression and psychological impairment persistence, in spite of excellent weight reduction. These observations contrast with publications in which depression and other Axis I disorders were associated with high pre-operative BMI, and with less weight reduction following bariatric surgery. ${ }^{21,22}$ From the results of the present study, one might speculate that the higher rates of comorbidity persistence in Caucasians may have exacerbated depression and other psychological impairments for those patients. Although the causes of these racial variations are not clear from the data, increased post-LRYGB Caucasian depression is a significant new observation.

Overall, more obesity comorbidities persisted following LRYGB among those of the Caucasian persuasion than in the other races. Caucasians had the greatest percentage of fourteen weight-related problems, including cholelithiasis, liver disease, diabetes, depression, and mental health diagnoses. Hispanics, on the other hand, experienced excellent BMI reduction, with resolution of the greatest number of comorbidities, including GERD, dyslipidemia, back pain, depression, and eight others. While Asians had the highest prevalence of liver disease, diabetes, gout, dyslipidemia, mental health diagnosis, and alcohol consumption, they resolved six other categories at the highest rates. African-Americans and Other performed intermediate between Caucasians and Hispanics. These variations in post-LRYGB outcomes according to race have not been reported previously in the literature. ${ }^{10,15,22,23}$

There are several limitations to this study. Firstly, the limits of a retrospective analysis of a prospectively collected database apply. Secondly, only clinical definitions were used in BOLD, including 
liver disease, where biopsies were not required. Thirdly, after 24 months, analyses had reduced statistical power due to declining follow-up visits. Fourthly, the patients here chose to have LRYGB, and therefore the results of this study might not be applicable to other bariatric operations. While baseline data was included in the statistical analyses, pre-operative racial variations still may have influenced temporally downstream results. Finally, having to group the BOLD sub-categories of severity for obesity comorbidities together for statistical robustness precluded a more in-depth evaluation of these conditions.

\section{Conclusion}

Post-operative weight, BMI and the resolution of weight-related medical problems among morbidly obese patients who undergo LRYGB vary according to racial classifications. Weight and BMI persisted highest for African Americans and Asians. Hispanics benefitted most from LRYGB in the greatest number of obesity comorbidities. Caucasians resolved these conditions least frequently. African-Americans, Asians, and the Other grouping outcomes were a middle cohort. While some racial variations persisted only six months, the advance knowledge of LRYGB outcomes variation by race presented here may help optimize management of morbidly obese patients who choose this operation.

\section{Acknowledgments}

None.

\section{Conflicts of interest}

The authors declare that they have no conflict of interest.

\section{Funding}

None.

\section{References}

1. Pratt GM, Learn CA, Hughes GD, et al. Demographics and outcomes at American Society for Metabolic and Bariatric Surgery Centers of Excellence. Surg Endosc Other Interv Tech. 2009;23(4):795-799.

2. Frank ATH, Zhao B, Jose PO, et al. Racial/ethnic differences in dyslipidemia patterns. Circulation. 2014;129(5):570-579.

3. Balasubramanian G, Singh M, Gupta N, et al. Prevalence and predictors of columnar lined esophagus in gastroesophageal reflux disease (GERD) patients undergoing upper endoscopy. Am $J$ Gastroenterol. 2012;107(11):1655-1661.

4. Maynard JW, McAdams-Demarco MA, Law A, et al. Racial differences in gout incidence in a population-based cohort: Atherosclerosis risk in communities study. Am J Epidemiol. 2014;179(5):576-583.

5. Gara MA, Vega WA, Arndt S, et al. Influence of patient race and ethnicity on clinical assessment in patients with affective disorders. Arch Gen Psychiatry. 2012;69(6):593-600.

6. Knox JB, Orchowski JR, Owens B. Racial differences in the incidence of acute low back pain in United States military service members. Spine (Phila Pa 1976). 2012;37(19):1688-1692.
7. Townsend MK, Curhan GC, Resnick NM, et al. The incidence of urinary incontinence across Asian, black, and white women in the United States. Am J Obstet Gynecol. 2010;202(4):378.e1-378.e7.

8. Shield KD, Gmel G, Kehoe-Chan T, et al. Mortality and Potential Years of Life Lost Attributable to Alcohol Consumption by Race and Sex in the United States in 2005. PLoS One. 2013;8(1).

9. DeMaria EJ, Pate V, Warthen M, et al. Baseline data from American society for metabolic and bariatric surgery-designated bariatric surgery centers of excellence using the bariatric outcomes longitudinal database. Surg Obes Relat Dis. 2010;6(4):347-355.

10. Khorgami Z, Arheart KL, Zhang C, et al. Effect of Ethnicity on Weight Loss After Bariatric Surgery. Comparative Study. 2015;769-776.

11. Bayham BE, Bellanger DE, Hargroder AG, et al. Racial Differences in Weight Loss, Payment Method, and Complications Following Rouxen-Y Gastric Bypass and Sleeve Gastrectomy. 2012;29:970-978.

12. Limbach KE, Ashton K, Merrell J, et al. Relative Contribution of Modifiable Versus Non-Modifiable Factors as Predictors of Racial Variance in Roux-en-Y Gastric Bypass Weight Loss Outcomes. Obes Surg. 2014;24(8):1379-1385.

13. Winegar D, Thomas S. Influence of Ethnicity on the Efficacy and Utilization of Bariatric Surgery in the USA. 2014;130-136.

14. Cheung LK, Lal LS, Chow DS, Sherman V. Racial Disparity in ShortTerm Outcomes after Gastric Bypass Surgery. 2013;2008:2096-103.

15. Coleman KJ, Brookey J. Gender and Racial / Ethnic Background Predict Weight Loss after Roux-en-Y Gastric Bypass Independent of Health and Lifestyle Behaviors. 2014;1729-1736.

16. Coleman KJ, Huang Y, Koebnick C, et al. Metabolic Syndrome Is Less Likely to Resolve in Hispanics and Non-Hispanic Blacks After Bariatric Surgery. 2014;259(2):279-285.

17. Ng J, Ph D, Seip R, et al. Ethnic variation in weight loss, but not comorbidity remission, after laparoscopic gastric banding and Roux-en-Y gastric bypass. Surg Obes Relat Dis. 2015;11(1):94-100.

18. Gullick AA, Graham LA, Richman J, et al. Association of Race and Socioeconomic Status with Outcomes Following Laparoscopic Rouxen-Y Gastric Bypass. Obes Surg. 2014;25(4):705-711.

19. Blankfield RP, Hudgel DW, Tapolyai A, et al. Bilateral leg edema, obesity, pulmonary hypertension, and obstructive sleep apnea. Arch Intern Med. 2000;160(15):2357-2362.

20. Birn I, Mechlenburg I, Liljensøe A, et al. The Association Between Preoperative Symptoms of Obesity in Knee and Hip Joints and the Change in Quality of Life After Laparoscopic Roux-en-Y Gastric Bypass. Obes Surg. 2015;1-7.

21. Hammerton G, Thapar A, Thapar a K. Association between obesity and depressive disorder in adolescents at high risk for depression. Int $J$ Obes. 2013;38(4):513-519.

22. Messiah SE, Lopez-mitnik G, Winegar D, et al. Effect of ethnicity on weight loss among adolescents 1 year after bariatric surgery. 2013;4(5):202-209. 